Kragujevac Journal of Mathematics

Volume 39(1) (2015), Pages 83-91.

\title{
SOME INEQUALITIES FOR CONVEX AND 3-CONVEX FUNCTIONS WITH APPLICATIONS
}

\author{
DAN ŞTEFAN MARINESCU ${ }^{1}$ AND MIHAI MONEA ${ }^{2}$
}

\begin{abstract}
In this paper we prove some new inequalities involving the $n$-convex functions, where $n \in\{2,3\}$.
\end{abstract}

\section{INTRODUCTION}

In [1], G. Bennett presented some consequences of an inequality describing the behavior of convex functions with respect to a mass distribution. Later, C. P. Niculescu proved an abstract version of this result([9]), which is shown in the next theorem.

Theorem 1.1. Suppose that I is an interval carrying a positive Borel measure $\mu$ and $A, B, C$ are three nonoverlapping compact subintervals of $I$ of positive measure. Then

$$
\mu(B)=\mu(A)+\mu(C)
$$

and

$$
\int_{B} t d \mu(t)=\int_{A} t d \mu(t)+\int_{C} t d \mu(t)
$$

provide a set of necessary and sufficient conditions under which every convex function $f: I \rightarrow \mathbb{R}$ verifies the inequality

$$
\int_{B} f(t) d \mu(t) \leq \int_{A} f(t) d \mu(t)+\int_{C} f(t) d \mu(t) .
$$

The aim of this paper is to present similar results for the Riemann integral. We establish some conditions for the validity of some integral inequality involving the convex functions. The main results are described in the Theorem 2.1 and Theorem 2.2 from the next section. Like consequences, we obtain new characterizations of the convex functions (see Theorem 2.3). In the third section, we prove some results

Key words and phrases. $n$-convex function, inequality, Riemann integral.

2010 Mathematics Subject Classification. Primary: 26A51. Secondary: 26D15, 26A42.

Received: May 19, 2014

Accepted: February 26, 2015. 
of same type for 3-convex functions, including the Theorem 2 from G. Bennett's $\operatorname{paper}([1])$.

\section{Some inequalities InVOLVING CONVEX FUnCTiOnS}

We start this section with some considerations about the convex functions. We denote $I$, a real interval and denote $K_{2}(I)$, the set of the convex functions $f: I \rightarrow \mathbb{R}$. It is knowing that every $f \in K_{2}(I)$ is Riemann integrable on any compact interval $[a, b] \subset I$ (see Proposition 1.3.4, [8]). Furthermore, for every $x_{0} \in I$, the function

$$
s: I \backslash\left\{x_{0}\right\} \rightarrow \mathbb{R}, s(x)=\frac{f(x)-f\left(x_{0}\right)}{x-x_{0}}
$$

is non-decreasing (see Proposition 1.3.1, [8]).

To avoid the repetitions, we establish some conditions and notations which we will use it in all of this paper. Let be $a_{1}, a_{2}, b_{1}, b_{2}, c_{1}, c_{2} \in I$ such that

$$
a_{1}<a_{2} \leq b_{1}<b_{2} \leq c_{1}<c_{2} .
$$

Denote $a=a_{1}+a_{2}, b=b_{1}+b_{2}$ and $c=c_{1}+c_{2}$.

Theorem 2.1. Let $p, q, r$ be real positive numbers. The next inequality

$$
q \cdot \frac{f\left(b_{2}\right)+f\left(b_{1}\right)}{2} \leq p \cdot \frac{\int_{a_{1}}^{a_{2}} f(x) d x}{a_{2}-a_{1}}+r \cdot \frac{\int_{c_{1}}^{c_{2}} f(x) d x}{c_{2}-c_{1}},
$$

holds for every function $f \in K_{2}(I)$ if and only if $q=p+r$ and $q \cdot b=p \cdot a+r \cdot c$.

Proof. For the 'if' part, we choose the functions $f_{1}, f_{2}, f_{3}, f_{4}: I \rightarrow \mathbb{R}$, defined by $f_{1}(x)=1, f_{2}(x)=-1, f_{3}(x)=x$ and $f_{4}(x)=-x$, for every $x \in I$. Then $f_{1}, f_{2}, f_{3}, f_{4} \in$ $K_{2}(I)$. We apply the inequality (2.1) for $f_{1}$ and we obtain $q \leq p+r$. The function $f_{2}$ and the inequality $(2.1)$ goes to $-q \leq-p-r$ and we find $q=p+r$.

In same mode, the inequality $(2.1)$ and the function $f_{3}$ give us the inequality $q \cdot b \leq p \cdot a+r \cdot c$. We apply inequality (2.1) for $f_{4}$ and we obtain $-q \cdot b \leq-p \cdot a-r \cdot c$. Now, the conclusion follows.

For the 'only if' part, we note that the equalities $q=p+r$ and $q \cdot b=p \cdot a+r \cdot c$ are equivalent with

$$
\frac{p}{c-b}=\frac{q}{c-a}=\frac{r}{b-a} .
$$

Further, the inequality (2.1) is equivalent to

$$
(c-a) \cdot \frac{f\left(b_{2}\right)+f\left(b_{1}\right)}{2} \leq(c-b) \cdot \frac{\int_{a_{1}}^{a_{2}} f(x) d x}{a_{2}-a_{1}}+(b-a) \cdot \frac{\int_{c_{1}}^{c_{2}} f(x) d x}{c_{2}-c_{1}} .
$$

Since $f \in K_{2}(I)$, the function $s: I \backslash\left\{b_{1}\right\} \rightarrow \mathbb{R}, s(x)=\frac{f(x)-f\left(b_{1}\right)}{x-b_{1}}$ is non-decreasing. Then

$$
\frac{f(x)-f\left(b_{1}\right)}{x-b_{1}} \leq \frac{f\left(b_{2}\right)-f\left(b_{1}\right)}{b_{2}-b_{1}}
$$


for any $x \in\left[a_{1}, a_{2}\right]$ and

$$
\frac{f(x)-f\left(b_{1}\right)}{x-b_{1}} \geq \frac{f\left(b_{2}\right)-f\left(b_{1}\right)}{b_{2}-b_{1}}
$$

for any $x \in\left[c_{1}, c_{2}\right]$.

The conclusion is

$$
f(x) \geq f\left(b_{1}\right)+\frac{f\left(b_{2}\right)-f\left(b_{1}\right)}{b_{2}-b_{1}}\left(x-b_{1}\right),
$$

for any $x \in\left[a_{1}, a_{2}\right] \cup\left[c_{1}, c_{2}\right]$. By integrating the last inequality on $\left[a_{1}, a_{2}\right]$ and also on $\left[c_{1}, c_{2}\right]$ and adding up the resulted inequalities, we obtain

$$
\begin{aligned}
& (c-b) \cdot \frac{\int_{a_{1}}^{a_{2}} f(x) d x}{a_{2}-a_{1}}+(b-a) \cdot \frac{\int_{c_{1}}^{c_{2}} f(x) d x}{c_{2}-c_{1}} \\
& \geq f\left(b_{1}\right)(c-a)+\frac{1}{2} \cdot \frac{f\left(b_{2}\right)-f\left(b_{1}\right)}{b_{2}-b_{1}}\left[\left(a-2 b_{1}\right)(c-b)+\left(c-2 b_{1}\right)(b-a)\right] \\
& =f\left(b_{1}\right)(c-a)+\frac{1}{2} \cdot \frac{f\left(b_{2}\right)-f\left(b_{1}\right)}{b_{2}-b_{1}}(c-a)\left(b_{2}-b_{1}\right)=(c-a) \cdot \frac{f\left(b_{2}\right)+f\left(b_{1}\right)}{2},
\end{aligned}
$$

which shows that the inequality $(2.2)$ is true.

In the particular case, when $q=b_{2}-b_{1}, p=a_{2}-a_{1}$ and $r=c_{2}-c_{1}$, the equality $b_{2}-b_{1}=a_{2}-a_{1}+c_{2}-c_{1}$ becomes $q=p+r$. Moreover, the equality $b_{2}^{2}-b_{1}^{2}=a_{2}^{2}-a_{1}^{2}+c_{2}^{2}-c_{1}^{2}$ is equivalent with $q \cdot b=p \cdot a+r \cdot c$. Then, we obtain the next consequence.

Corollary 2.1. The inequality

$$
\left(b_{2}-b_{1}\right) \cdot \frac{f\left(b_{2}\right)+f\left(b_{1}\right)}{2} \leq \int_{a_{1}}^{a_{2}} f(x) d x+\int_{c_{1}}^{c_{2}} f(x) d x
$$

is true for any $f \in K_{2}(I)$ if and only if $b_{2}-b_{1}=a_{2}-a_{1}+c_{2}-c_{1}$ and $b_{2}^{2}-b_{1}^{2}=$ $a_{2}^{2}-a_{1}^{2}+c_{2}^{2}-c_{1}^{2}$.

A very useful result about convex functions is presented by Hadamard-Hermite inequality(for example, see Corollary 3.7.2, [3]). It says that for any function $f \in$ $K_{2}(I)$ and for any $a, b \in I$ such that $a<b$, we have

$$
f\left(\frac{a+b}{2}\right) \leq \frac{1}{b-a} \int_{a}^{b} f(x) d x \leq \frac{f(a)+f(b)}{2} .
$$

We use this tool to prove the following theorem.

Theorem 2.2. Let $p, q, r$ be real positive numbers. The inequality

$$
q \cdot \frac{\int_{b_{1}}^{b_{2}} f(x) d x}{b_{2}-b_{1}} \leq p \cdot \frac{\int_{a_{1}}^{a_{2}} f(x) d x}{a_{2}-a_{1}}+r \cdot \frac{\int_{c_{1}}^{c_{2}} f(x) d x}{c_{2}-c_{1}}
$$

is true for any $f \in K_{2}(I)$ if and only if $q=p+r$ and $q \cdot b=p \cdot a+r \cdot c$. 
Proof. The proof of the 'if' part is similar with the proof of same part of Theorem 2.1. For 'only if' part, we are using Theorem 2.1 and we have

$$
(c-a) \cdot \frac{f\left(b_{2}\right)+f\left(b_{1}\right)}{2} \leq(c-b) \cdot \frac{\int_{a_{1}}^{a_{2}} f(x) d x}{a_{2}-a_{1}}+(b-a) \cdot \frac{\int_{c_{1}}^{c_{2}} f(x) d x}{c_{2}-c_{1}} .
$$

By applying (2.3), we obtain

$$
\frac{\int_{b_{1}}^{b_{2}} f(x) d x}{b_{2}-b_{1}} \leq \frac{f\left(b_{2}\right)+f\left(b_{1}\right)}{2}
$$

Then

$$
(c-a) \cdot \frac{\int_{b_{1}}^{b_{2}} f(x) d x}{b_{2}-b_{1}} \leq(c-b) \cdot \frac{\int_{a_{1}}^{a_{2}} f(x) d x}{a_{2}-a_{1}}+(b-a) \cdot \frac{\int_{c_{1}}^{c_{2}} f(x) d x}{c_{2}-c_{1}} .
$$

Now, we find the conclusion in same mode like the proof of the Theorem 2.1 by using the equality

$$
\frac{p}{c-b}=\frac{q}{c-a}=\frac{r}{b-a} .
$$

In same particular case like to Corollary 2.1, we recover a result due to Niculescu $([9])$.

Corollary 2.2. The inequality

$$
\int_{b_{1}}^{b_{2}} f(x) d x \leq \int_{a_{1}}^{a_{2}} f(x) d x+\int_{c_{1}}^{c_{2}} f(x) d x
$$

holds for any $f \in K_{2}(I)$ if and only if $b_{2}-b_{1}=a_{2}-a_{1}+c_{2}-c_{1}$ and $b_{2}^{2}-b_{1}^{2}=$ $a_{2}^{2}-a_{1}^{2}+c_{2}^{2}-c_{1}^{2}$.

With some algebraic manipulation, Marinescu obtained a similar result ([6]) . It is following now.

Corollary 2.3. Let $f \in K_{2}(I)$ and let $a_{1}, a_{2}, \ldots, a_{2 n}, a_{2 n+1}$ be real numbers from $I$ in arithmetic progression with a positive ratio. Then

$$
\int_{a_{n}}^{a_{n+2}} f(x) d x \leq \int_{a_{1}}^{a_{2}} f(x) d x+\int_{a_{2 n}}^{a_{2 n+1}} f(x) d x .
$$

Proof. With $r$ we denote the ratio. It is clear that $a_{n+2}-a_{n}=a_{2}-a_{1}+a_{2 n+1}-a_{2 n}$. Now

$$
\begin{aligned}
a_{2}^{2}-a_{1}^{2}+a_{2 n+1}^{2}-a_{2 n}^{2} & =\left(a_{2}-a_{1}\right)\left(a_{2}+a_{1}\right)+\left(a_{2 n+1}-a_{2 n}\right)\left(a_{2 n+1}+a_{2 n}\right) \\
& =r\left(a_{2}+a_{1}\right)+r\left(a_{2 n+1}+a_{2 n}\right)=r\left(a_{2 n+1}+a_{2 n}+a_{2}+a_{1}\right) \\
& =r\left(\left(a_{2 n+1}+a_{1}\right)+\left(a_{2 n}+a_{2}\right)\right)=4 r a_{n+1} \\
& =2 r\left(a_{n+2}+a_{n}\right)=\left(a_{n+2}-a_{n}\right)\left(a_{n+2}+a_{n}\right) \\
& =a_{n+2}^{2}-a_{n}^{2} .
\end{aligned}
$$

We obtain the conclusion by applying the Corollary 2.2 . 
As known, when continuity is present, the Hadamard-Hermite inequalities characterizes the convexity (see Theorem 3.7.8. from [3]). Next we will establish some results of the same nature.

Theorem 2.3. Let be $f: I \rightarrow \mathbb{R}$ a continuous function. The next three statements are equivalent:

(i) The function $f$ is convex;

(ii) For any $a_{1}, a_{2}, b_{1}, b_{2}, c_{1}, c_{2} \in I$ such that $a_{1}<a_{2} \leq b_{1}<b_{2} \leq c_{1}<c_{2}$, we have

$$
(c-a) \cdot \frac{f\left(b_{2}\right)+f\left(b_{1}\right)}{2} \leq(c-b) \cdot \frac{\int_{a_{1}}^{a_{2}} f(x) d x}{a_{2}-a_{1}}+(b-a) \cdot \frac{\int_{c_{1}}^{c_{2}} f(x) d x}{c_{2}-c_{1}},
$$

where $a=a_{1}+a_{2}, b=b_{1}+b_{2}, c=c_{1}+c_{2}$;

(iii) For any $a_{1}, a_{2}, b_{1}, b_{2}, c_{1}, c_{2} \in I$ such that $a_{1}<a_{2} \leq b_{1}<b_{2} \leq c_{1}<c_{2}$, we have

$$
(c-a) \cdot \frac{\int_{b_{1}}^{b_{2}} f(x) d x}{b_{2}-b_{1}} \leq(c-b) \cdot \frac{\int_{a_{1}}^{a_{2}} f(x) d x}{a_{2}-a_{1}}+(b-a) \cdot \frac{\int_{c_{1}}^{c_{2}} f(x) d x}{c_{2}-c_{1}} .
$$

where $a=a_{1}+a_{2}, b=b_{1}+b_{2}, c=c_{1}+c_{2}$.

Proof. First, we prove the equivalence $(i) \Leftrightarrow(i i)$. The 'if' part is a particular case of Theorem 2.1.

For the 'only if' part, let $x, y \in I$ and $t \in(0,1)$. Denote $z=t x+(1-t) y$. We admit that $x<y$. Then we have $x<z<y$. We consider $\varepsilon>0$ so that

$$
x<x+\varepsilon<z-\varepsilon<z<y-\varepsilon<y .
$$

We choose $a_{1}=x, a_{2}=x+\varepsilon, b_{1}=z-\varepsilon, b_{2}=z, c_{1}=y-\varepsilon$ and $c_{2}=y$. Then, we have

$$
(2 y-2 x-2 \varepsilon) \cdot \frac{f(z)+f(z-\varepsilon)}{2} \leq(2 y-2 z) \cdot \frac{\int_{x}^{x+\varepsilon} f(t) d t}{\varepsilon}+(2 z-2 x-2 \varepsilon) \cdot \frac{\int_{y-\varepsilon}^{y} f(t) d t}{\varepsilon} .
$$

Since the function $f$ is continuous, then

$$
\lim _{\varepsilon \rightarrow 0} \frac{\int_{x}^{x+\varepsilon} f(t) d t}{\varepsilon}=f(x) .
$$

In (2.6), we put $\varepsilon \rightarrow 0$ and we obtain

$$
(y-x) \cdot f(z) \leq(y-z) \cdot f(x)+(z-x) \cdot f(y),
$$

also

$$
f(z) \leq t \cdot f(x)+(1-t) \cdot f(y) .
$$

This shows that the function $f$ is convex.

Now, we proceed in same mode for the equivalence $(i) \Leftrightarrow($ iii $)$. The 'if' part is a particular case of Theorem 2.2. The proof of the 'only if' part is similar, but we have the inequality

$$
(2 y-2 x-2 \varepsilon) \cdot \frac{\int_{z-\varepsilon}^{z} f(t) d t}{\varepsilon} \leq(2 y-2 z) \cdot \frac{\int_{x}^{x+\varepsilon} f(t) d t}{\varepsilon}+(2 z-2 x-2 \varepsilon) \cdot \frac{\int_{y-\varepsilon}^{y} f(t) d t}{\varepsilon}
$$


instead of (2.6). We put $\varepsilon \rightarrow 0$ and our proof is complete.

The implication $(i i i) \Rightarrow(i)$ can be found in [7], but the Theorem 2.3 give us a supplementary characterization for convexity due to the implication $(i i) \Rightarrow(i)$.

\section{Some Results ABout the 3-COnVEx FunCtions}

Let be $n \in \mathbb{N}^{\star}$. The notion of $n$-convex function is due to T. Popoviciu (see [11]). More details about these functions and their properties can be found in [4], [5] or [10]. In this section we focus to the case $n=3$. First, let be $I$ an open interval of $\mathbb{R}$. We recall that a function $f: I \rightarrow \mathbb{R}$ is 3-convex if

$$
\sum_{\text {cyclic }} \frac{1}{(x-y)(x-z)(x-t)} \cdot f(x) \geq 0,
$$

for any $x, y, z, t \in I$, with $x<y<z<t$.

We denote $K_{3}(I)$, the set of all the functions $f: I \rightarrow \mathbb{R}$ which is 3 -convex on $I$. Is is known that every function $f \in K_{3}(I)$ is derivable and $f^{\prime} \in K_{2}(I)$ (see Theorem 1.41 from [10]). Moreover, for any derivable function $f: I \rightarrow \mathbb{R}$ such that $f^{\prime} \in K_{2}(I)$, we have $f \in K_{3}(I)$ (see Theorem 15.8.2 from [5]).

Next, we obtain some results for 3-convex functions, similar to Theorem 2.1 or Theorem 2.2. We use same notation like in the previous section.

Theorem 3.1. Let be $f \in K_{3}(I)$ and $p, q, r$ be real positive numbers. The following inequality holds

$$
q \cdot \frac{f\left(b_{2}\right)-f\left(b_{1}\right)}{b_{2}-b_{1}} \leq p \cdot \frac{f\left(a_{2}\right)-f\left(a_{1}\right)}{a_{2}-a_{1}}+r \cdot \frac{f\left(c_{2}\right)-f\left(c_{1}\right)}{c_{2}-c_{1}}
$$

if and only if $q=p+r$ and $q \cdot b=p \cdot a+r \cdot c$.

Proof. The proof of the 'if' part is similar with same part of the Theorem 2.1, but we are using the functions $f_{1}, f_{2}, f_{3}, f_{4}: I \rightarrow \mathbb{R}$ defined by $f_{1}(x)=x, f_{2}(x)=-x$, $f_{3}(x)=x^{2}$ and $f_{4}(x)=-x^{2}$, for any $x \in I$. This functions are 3 -convex and similar calculus gives us $q=p+r$ and $q \cdot b=p \cdot a+r \cdot c$.

For the 'only if' part, first we note that the inequality (3.1) is equivalent with

$$
(c-a) \cdot \frac{f\left(b_{2}\right)-f\left(b_{1}\right)}{b_{2}-b_{1}} \leq(c-b) \cdot \frac{f\left(a_{2}\right)-f\left(a_{1}\right)}{a_{2}-a_{1}}+(b-a) \cdot \frac{f\left(c_{2}\right)-f\left(c_{1}\right)}{c_{2}-c_{1}} .
$$

Since $f \in K_{3}(I)$, then $f$ is derivable and $f^{\prime} \in K_{2}(I)$. Now, we apply Theorem 2.2 for the function $f^{\prime}$ and we obtain the inequality (3.2) and the conclusion.

By applying Theorem 3.1 for $q=b_{2}-b_{1}, p=a_{2}-a_{1}$ and $r=c_{2}-c_{1}$, we obtain the next corollary, similar with Corollary 2.1.

Corollary 3.1. Let be $f \in K_{3}(I)$. Then

$$
f\left(b_{2}\right)+f\left(a_{1}\right)+f\left(c_{1}\right) \leq f\left(b_{1}\right)+f\left(a_{2}\right)+f\left(c_{2}\right)
$$

if and only if $b_{2}-b_{1}=a_{2}-a_{1}+c_{2}-c_{1}$ and $b_{2}^{2}-b_{1}^{2}=a_{2}^{2}-a_{1}^{2}+c_{2}^{2}-c_{1}^{2}$. 
The result from the next corollary represents the Theorem 2 from [1]. For its proof we need the following lemma.

Lemma 3.1. Let be $m, n, p, u, v, w \in \mathbb{R}$ such that $m \leqslant n \leqslant p$ and $u \leqslant v \leqslant w$. If

$$
\begin{gathered}
m+n+p=u+v+w, \\
m^{2}+n^{2}+p^{2}=u^{2}+v^{2}+w^{2}
\end{gathered}
$$

and $p \leqslant w$, then $m \leqslant u$.

Proof. From (3.3) and (3.4), we obtain $m n+n p+p m=u v+v w+w u$. Denote $A=m+n+p$ and $B=m n+n p+p m$. We consider the functions $f, g: \mathbb{R} \rightarrow \mathbb{R}$, defined for any $x \in \mathbb{R}$ by

$$
f(x)=(x-m)(x-n)(x-p)
$$

and

$$
g(x)=(x-u)(x-v)(x-w) .
$$

Then, there exist $C, D \in \mathbb{R}$ so that

$$
f(x)=x^{3}-A x^{2}+B x+C
$$

and

$$
g(x)=x^{3}-A x^{2}+B x+D .
$$

Now is clear that $f(x)-g(x)=C-D$, for any $x \in \mathbb{R}$.

Further, $f(x) \geqslant 0$ for any $x \in[p, \infty)$. Then $f(w) \geqslant 0=g(w)$ and we obtain $C \geqslant$ $D$. In same time, we have $g(x) \leqslant 0$, for any $x \in(-\infty, u]$. Then $0=f(m) \geqslant g(m)$, which concludes our proof.

We can remark that the proof of previous lemma shows us that we have two distinct situations. If $p=w$, then $m=u$ and $n=v$. If $p<w$, we obtain $m<u$ and we have

$$
m<u \leq v<n \leq p<w .
$$

Corollary 3.2. Let $m, n, p, u, v, w$ be real numbers. Then the inequality

$$
f(m)+f(n)+f(p) \leq f(u)+f(v)+f(w)
$$

holds for any $f \in K_{3}(\mathbb{R})$ if and only if

$$
\begin{gathered}
m+n+p=u+v+w, \\
m^{2}+n^{2}+p^{2}=u^{2}+v^{2}+w^{2}
\end{gathered}
$$

and $\max \{m, n, p\} \leq \max \{u, v, w\}$.

Proof. We use the functions $f_{1}, f_{2}, f_{3}, f_{4}: I \rightarrow \mathbb{R}$ defined by $f_{1}(x)=x, f_{2}(x)=-x$, $f_{3}(x)=x^{2}$ and $f_{4}(x)=-x^{2}$ for any $x \in I$ and we obtain the proof of the 'if' part .

For the 'only if' part, we can admit that $m \leq n \leq p$ and $u \leq v \leq w$. Now, the inequality $\max \{m, n, p\} \leq \max \{u, v, w\}$ becames $p \leq w$. Lemma 3.1 shows us that $m \leq u$. Due to the previous remark, two cases are possible. If $\{m, n, p\}=\{x, y, z\}$, 
then the conclusion is trivial. If $p<w$, the conclusion is consequence of the Corollary 3.1 .

We conclude this paper with two characterizations of the 3 -convex function, similar to Theorem 2.3.

Theorem 3.2. Let be $f: I \rightarrow \mathbb{R}$ a continuously differentiable function. Then $f \in$ $K_{3}(I)$ if and only if for any $a_{1}, a_{2}, b_{1}, b_{2}, c_{1}, c_{2} \in I$, such that $a_{1}<a_{2} \leq b_{1}<b_{2} \leq$ $c_{1}<c_{2}$, the next inequality holds

$$
(c-a) \cdot \frac{f^{\prime}\left(b_{1}\right)+f^{\prime}\left(b_{2}\right)}{2} \leq(c-b) \cdot \frac{f\left(a_{2}\right)-f\left(a_{1}\right)}{a_{2}-a_{1}}+(b-a) \cdot \frac{f\left(c_{2}\right)-f\left(c_{1}\right)}{c_{2}-c_{1}},
$$

where $a=a_{1}+a_{2}, b=b_{1}+b_{2}$ and $c=c_{1}+c_{2}$.

Proof. The relation (3.5) can be written as

$$
(c-a) \cdot \frac{f^{\prime}\left(b_{1}\right)+f^{\prime}\left(b_{2}\right)}{2} \leq(c-b) \cdot \frac{\int_{a_{1}}^{a_{2}} f^{\prime}(x) d x}{a_{2}-a_{1}}+(b-a) \cdot \frac{\int_{c_{1}}^{c_{2}} f^{\prime}(x) d x}{c_{2}-c_{1}} .
$$

Now, we obtain the conclusion by using the equivalence $(i) \Leftrightarrow(i i)$ from Theorem 2.3, applied for the continuous function $f^{\prime}$.

Theorem 3.3. Let $f: I \rightarrow \mathbb{R}$ be a differentiable function. Then $f \in K_{3}(I)$ if and only if for any $a_{1}, a_{2}, b_{1}, b_{2}, c_{1}, c_{2} \in I$, such that $a_{1}<a_{2} \leq b_{1}<b_{2} \leq c_{1}<c_{2}$, the next inequality holds

$$
(c-a) \cdot \frac{f\left(b_{2}\right)-f\left(b_{1}\right)}{b_{2}-b_{1}} \leq(c-b) \cdot \frac{f\left(a_{2}\right)-f\left(a_{1}\right)}{a_{2}-a_{1}}+(b-a) \cdot \frac{f\left(c_{2}\right)-f\left(c_{1}\right)}{c_{2}-c_{1}},
$$

where $a=a_{1}+a_{2}, b=b_{1}+b_{2}$ and $c=c_{1}+c_{2}$.

Proof. If $f \in K_{3}(I)$, then $f^{\prime} \in K_{2}(I)$. By applying the implications $(i) \Rightarrow$ (iii) of Theorem 2.3 for the function $f^{\prime}$, we obtain the proof of the 'if' part.

For the 'only if' part, let $x, y \in I$ and $t \in(0,1)$. Denote $z=t x+(1-t) y$. We admit that $x<y$. Then we have $x<z<y$. We consider $\varepsilon>0$ so that

$$
x<x+\varepsilon<z-\varepsilon<z<y-\varepsilon<y .
$$

We choose $a_{1}=x, a_{2}=x+\varepsilon, b_{1}=z-\varepsilon, b_{2}=z, c_{1}=y-\varepsilon$ and $c_{2}=y$. Then, we have

$$
\begin{aligned}
(2 y-2 x-2 \varepsilon) \cdot \frac{f(z)-f(z-\varepsilon)}{\varepsilon} \leq & (2 y-2 z) \cdot \frac{f(x+\varepsilon)-f(x)}{\varepsilon} \\
& +(2 z-2 x-2 \varepsilon) \cdot \frac{f(y)-f(y-\varepsilon)}{\varepsilon} .
\end{aligned}
$$

By using the definition of a derivative, we obtain that $\lim _{\varepsilon \rightarrow 0} \frac{f(z)-f(z-\varepsilon)}{\varepsilon}=f^{\prime}(z)$, $\lim _{\varepsilon \rightarrow 0} \frac{f(x+\varepsilon)-f(x)}{\varepsilon}=f^{\prime}(x)$ and $\lim _{\varepsilon \rightarrow 0} \frac{f(y)-f(y-\varepsilon)}{\varepsilon}=f^{\prime}(y)$. In (3.7), we put $\varepsilon \rightarrow 0$ and we obtain

$$
(y-x) \cdot f^{\prime}(z) \leq(y-z) \cdot f^{\prime}(x)+(z-x) \cdot f^{\prime}(y),
$$


also

$$
f^{\prime}(z) \leq t \cdot f^{\prime}(x)+(1-t) \cdot f^{\prime}(y)
$$

which shows that the function $f^{\prime} \in K_{2}(I)$. Then $f \in K_{3}(I)$ and our proof is complete.

\section{REFERENCES}

[1] G. Bennett, p-free $l^{P}$ Inequalities, Amer. Math. Month. 117 (4) (2010), 334-351.

[2] R. P. Boas and D. V. Widder, Functions with positive differences, Duke Math. J. 7 (1940) 496-503.

[3] W. W. Breckner and T. Trif, Convex Functions and Related Functional Equations, Selected Topics, Cluj University Press, 2008, Cluj.

[4] P. S. Bullen, A criterion for n-convexity, Pacific Journal of Mathematics, 36 (1) (1971), 81-98.

[5] M. Kuczma, An introduction to the Theory of Functionals Equations and Inequalities, Second Edition, Birkhauser, 2009, Basel-Boston-Berlin.

[6] D. Ş. Marinescu, Generalizări ale unor probleme de convexitate, Gazeta Matematică, 3 (1994), 100-102.

[7] D. Ş. Marinescu, M. Monea, M. Stroe and M. Opincariu, Some new characterizations of the convex functions, Kragujevac J. Math. 2 (2013), 349-356.

[8] C. P. Niculescu and L. E. Persson, Convex Functions and Their Applications. A Contemporary Approach, Springen-Verlay, 2006, New York.

[9] C. P. Niculescu, On result of G. Bennett, Bull. Math. Soc. Sci. Math. Roumanie 54 (102) No. 3, (2011), 261-267.

[10] J. E. Pećarić, F. Proschan and Y. C. Tong, Convex Functions, Partial Orderings and Statistical Applications, Academic Press, 1992, New York.

[11] T. Popoviciu, Notes sur les fonctions convexes d'ordre superieur (IX), Bull. Math. Soc. Roum. Sci. 43 (1941), 85-141.

${ }^{1}$ National College "Iancu de Hunedoara", Hunedoara, Romania

E-mail address: marinescuds@gmail.com

${ }^{2}$ National College "Decebal",

Deva, Romania

E-mail address: mihaimonea@yahoo.com 